\title{
Chromatin in 3D: progress and prospects for plants
}

\section{Chang Liu ${ }^{*}$ and Detlef Weigel}

Abstract
Methods that use high-throughput sequencing have
begun to reveal features of the three-dimensional
structure of genomes at a resolution that goes far
beyond that of traditional microscopy. Integration of
these methods with other molecular tools has advanced
our knowledge of both global and local chromatin
packing in plants, and has revealed how patterns of
chromatin packing correlate with the genomic and
epigenomic landscapes. This update reports recent
progress made in this area in plants, and suggests new
research directions.

\section{Introduction}

Some time ago, cytological studies not only showed that chromosomes are arranged in species-specific ways during interphase but also suggested that chromosome length is an important determinant of overall chromosome conformation in the nucleus [1]. Some plant species have chromosomes that are several hundred megabases long, and these are often found in the 'Rabl' configuration [2], with centromeres and telomeres at opposite poles of the nucleus [3]. By contrast, short chromosomes tend to be arranged in a rosette configuration, such that the chromosome arms loop out from chromocenters that contain the densely packed centromeres [4]. In the model plant Arabidopsis thaliana, which has a small genome with chromosome arms of around 10 megabases, the positioning of genes within the nucleus can affect their expression levels [5]. The physical clustering of alleles that has been observed in A. thaliana [6] also suggests that genes are not randomly arranged within the chromosomes.

In non-plant species, the application of a 3C (chromatin conformation capture)-based method named $\mathrm{Hi}$-C has greatly advanced our understanding of genome packing. In

\footnotetext{
* Correspondence: chang.liu@tuebingen.mpg.de; weigel@weigelworld.org
Department of Molecular Biology, Max Planck Institute for Developmental

* Correspondence: chang.liu@tuebingen.mpg.de; weigel@weigelworld.org
Department of Molecular Biology, Max Planck Institute for Developmental Biology, Tübingen, Germany
}

particular, Hi-C has revealed that TADs (topologically associating domains) are a predominant structural feature in most organisms [7-12]. Each TAD is a relatively isolated local unit, such that chromatin contacts within one TAD are generally preferred over those between different TADs. The locations of TAD boundaries are strongly correlated with local gene expression, epigenetic landscape and, where this had been tested, the binding of various insulator proteins (reviewed in $[13,14]$ ). Here, we summarize what was previously known about nuclear chromosome arrangement in plants, and discuss how the recent application of $3 \mathrm{C}$ and related methods has provided a more detailed picture of chromatin packing in plants.

\section{Importance of local three-dimensional structure: chromatin loops}

Within the DNA sequence of a chromosome, transcriptional enhancers can often be located far away from transcription units, but chromatin loops can bring distal regulatory elements into direct contact with the promoters that they control. The first plant chromatin loop to be found using the $3 \mathrm{C}$ method was at the maize $b 1$ gene, which controls pigmentation. Several additional loops have recently been found at four $A$. thaliana genes that have oles in flowering and hormone signaling [15-20].

The $b 1$ gene in maize encodes a transcription factor that regulates anthocyanin pigment production. Two epialleles of the $b 1$ gene, $B-I$ and $B^{\prime}$, are distinguished by their level RNA expression, which is much higher in $B-I$ than in $B^{\prime}$. husk tissues specifically, both epialleles form chromatin ops between the gene body and an enhancer located 100 upstream [15]. In the $B-I$ allele, this enhancer has open ctructure between the $b 1$ transcription start site (ISS) and additional upstream regions that mediates high expression. By contrast, the $B$ enhancer has compact of $A$. thaliana has emerged as an ideal platform for the discovery of many epigenetic regulatory mechanisms, involving histone modifications, small RNAs and long non- 
coding RNAs [21]. In recent work, the $5^{\prime}$ end of the FLC transcribed region was found to interact with sequences immediately downstream of the polyadenylation signal [16]. In non-plant organisms, chromatin loops connecting the $5^{\prime}$ and $3^{\prime}$ ends of genes have been proposed to support transcription by facilitating the recycling of RNA polymerase at actively transcribed genes [22], or by promoting the recruitment of RNA polymerase to reactivate gene expression [23]. Although the loop at the FLC gene is comparable to examples outside plants, its formation does not directly correlate with RNA expression: it can be detected in a wide range of genetic backgrounds that have very different levels of $F L C$ expression. Its disruption does appear, however, to be an early response to prolonged cold exposure, or vernalization, which ultimately causes stable, Polycomb-mediated repression of FLC [16]. Disruption of the FLC loop relies on SWI/SNF chromatin-remodeling activity, as inferred from knockdown of the SWI-SNF subunit BAF60, which stabilizes the FLC chromatin loop [24].

Regulation of $F L C$ involves the long noncoding RNA (lncRNA) COOLAIR [25]. It has been suggested that disruption of the $F L C$ chromatin loop allows access of RNA polymerase II or of transcription factors to COOLAIR regulatory elements, which (through an intricate series of further events) ultimately leads to Polycomb-mediated silencing of FLC [16]. This scenario is similar to that proposed for the PINOID (PID) auxin-response gene, which has a chromatin loop in its promoter region [20]. This loop appears to preclude the binding of activating transcription factors, and with the loop present, PID expression is low. Formation of the loop depends on LHP1, an H3K27me3binding plant homolog of HETEROCHROMATIN PROTEIN $1[26,27]$. The promoter-distal end of the loop is densely methylated, and it contains the TSS of a lncRNA, APOLO (AUXIN-REGULATED PROMOTER LOOP), which is divergently transcribed relative to PID. Auxin stimulation induces DNA demethylation at the $A P O L O$ locus, which reduces the frequency of H3K27me3 marks and consequently LHP1 binding, and thereby leading to the opening of the loop. Simultaneously, APOLO expression is activated, which ultimately triggers a new round of RNA-dependent DNA methylation (RdDM) and deposition of associated H3K27me3 chromatin marks that repress the expression of PID. The full model thus suggests an elegant mechanism for transient induction of PID: auxin induces DNA demethylation, leading to ejection of LHP1 and disruption of the chromatin loop and thus activation of PID expression. Because APOLO lncRNA expression is activated at the same time, a new round of RdDM is initiated, leading to PID downregulation.

The flowering gene FLOWERING LOCUS T $(F T)$ integrates many different environmental cues, including vernalization (by virtue of being a direct $F L C$ target), photoperiod, age and ambient temperature. Much of this integration occurs at the $F T$ locus itself, and its regulatory sequences are accordingly complex, as is the pattern of $F T$ expression during the life cycle of the plant (reviewed in [28]). Sequences both upstream and downstream of the transcription unit, as well as intergenic elements, contribute to $F T$ transcriptional regulation. One of these elements, an enhancer located $5.3 \mathrm{~kb}$ upstream of the transcribed region, makes contact with the TSS $[18,19]$. This enhancer contains a CCAAT motif, which is typically bound by Nuclear Factor Y (NF-Y) transcription factors (also known as HAP, AnCF or CBF proteins). Some NF-Y proteins have been shown to bind to CONSTANS (CO) [29], a B-box factor that interacts with the element near the TSS and activates FT expression [30]. These results illustrate a typical scenario in which chromatin looping is a consequence of the interaction of enhancer-regulatory factor complexes with promoter-proximal sequences [18]. The FT homolog TERMINAL FLOWER 1 (TFL1) is regulated very differently than $F T$, but also has complex regulatory sequences that include an enhancer located downstream of the transcription unit [31]. Binding of this enhancer by a complex of MADS-domain transcription factors causes it to dissociate from the TSS, and in turn, the disappearance of this loop appears to cause reduced expression of TFL1 [17].

\section{Global chromatin packing in plants}

Like studies of animals and humans, the plant field has begun to go beyond the analysis of chromatin loops at individual loci and is rapidly adopting the $4 \mathrm{C}$ and $\mathrm{Hi}-\mathrm{C}$ genome-wide methods [32-35]. At the chromosomal level, $\mathrm{Hi}-\mathrm{C}$ maps generated from $A$. thaliana seedlings have revealed patterns that correspond well with cytological observations (Fig. 1).

At the megabase scale, the $A$. thaliana chromosome arms are partitioned into structural domains that can be classified as either compact or loose [33]. The correlation of this domain structure with the epigenetic landscape is partly reminiscent of that of the 'AB compartment' originally discovered in human $\mathrm{Hi}-\mathrm{C}$ data [36]. Formation of these largescale domains is unaffected in the crwn1 (crowded nuclei 1) and crwn 4 mutants, which both have much smaller nuclei than wild-type plants, suggesting independence between nuclear morphology and chromosome packing [33]. At a more local scale, $A$. thaliana differs from most other organisms in that obvious TADs are not a predominant feature of $A$. thaliana genome organization. The lack of animal-like TADs in A. thaliana correlates with the absence of homologs of canonical insulator proteins such as CTCF [32, 34]. Moreover, experiments with transgenes have revealed very little, if any, credible evidence for insulator-like DNA sequences in A. thaliana [37]. Nevertheless, analysis of a high-resolution A. thaliana Hi-C map led to the identification of over 1000 TAD-boundary-like and insulator-like regions [34]. These 


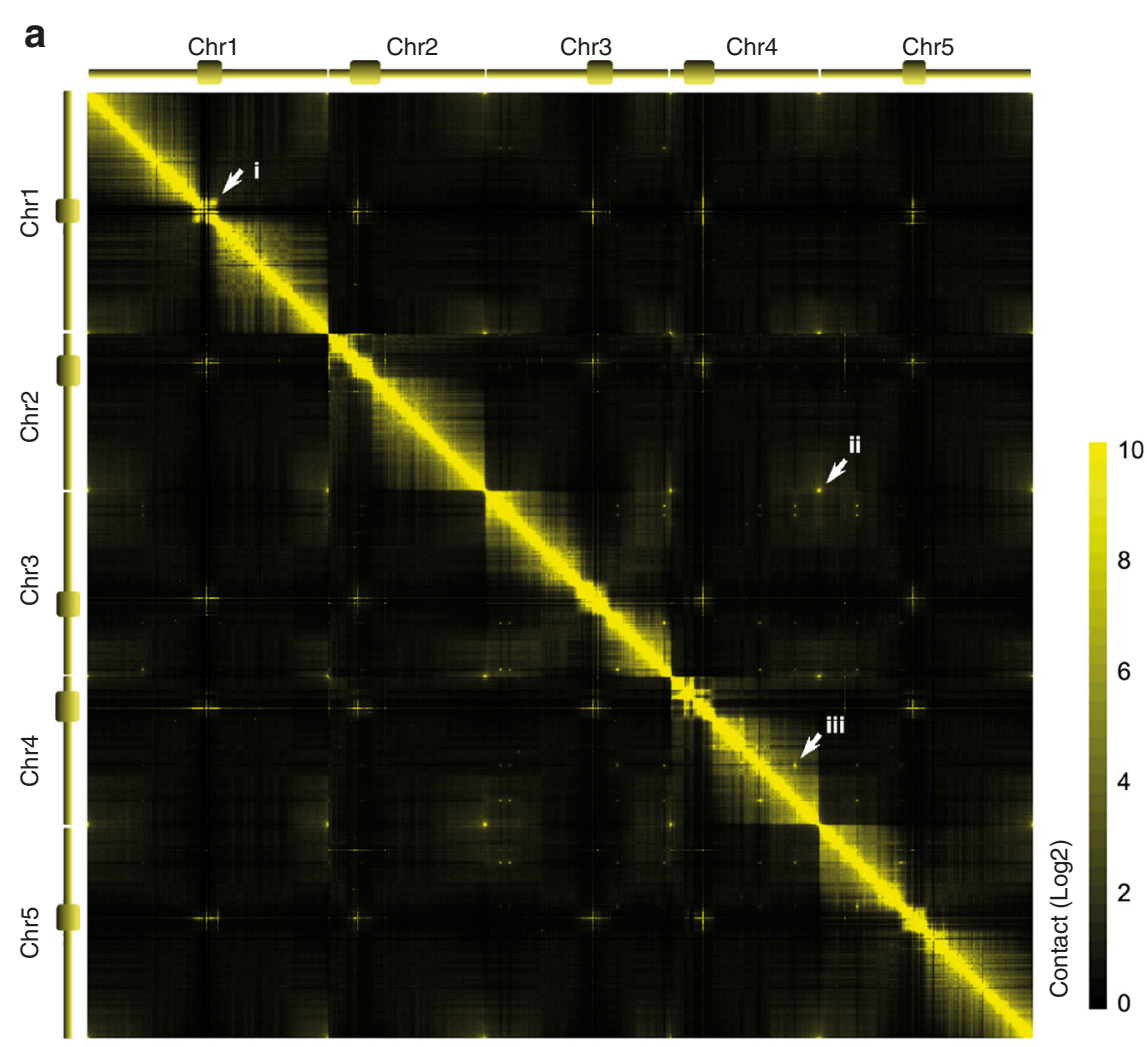

b
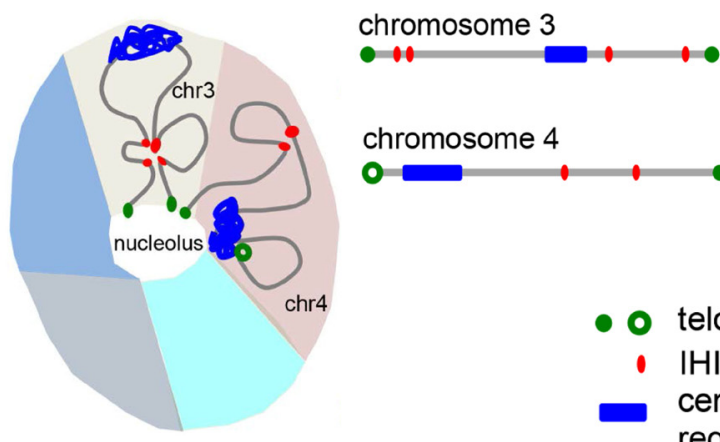

chromosome 4

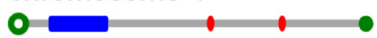

- telomere

- IHIs/KEEs

centromeric/pericentromeric regions

Fig. 1 Chromosome packing in interphase nuclei of Arabidopsis thaliana seedlings. a A genome-wide interaction map of A. thaliana seedlings at 20-kb resolution. The normalized contact strength is shown with color gradient. For all chromosomes, the intra-chromosomal contact decreases as a function of genomic distance. Arrows highlight selected features. (i) The centromeric and pericentromeric regions are tightly packed and have few interactions with regions located on chromosome arms. This corresponds to general observations that centromeric regions appear as bright dots in $A$. thaliana nuclei stained with DAPI (4'6-diamidino-2-phenylindole dihydrochloride). On the other hand, in $\mathrm{Hi}-\mathrm{C}$ maps of mutant plants where heterochromatin is decondensed, such as met1, ddm1, suvh4, suvh5 suvh6, and atmorc6, pericentromeric regions interact less with each other and more with the chromosome arms [32, 39]. (ii) Telomeres from different chromosomes are close to each other. According to fluorescent in situ hybridization (FISH) studies, telomeres often gather around the nucleolus [4]. (iii) Strong interaction among a subset of interstitial heterochromatin regions. These interactions have been captured by four independent Hi-C experiments [32-34, 39]; some of these interactions have also been seen with FISH [32, 33]. This $\mathrm{Hi}-\mathrm{C}$ map is reproduced from our previously published interaction matrix [34]. b Diagram summarizing the conformation of interphase A. thaliana chromosomes with packing inferred from cytological and $\mathrm{Hi}-\mathrm{C}$ experiments. Further details are shown for chromosomes 3 and 4 . Every chromosome occupies a distinct territory (five differently colored territories are shown in this nucleus cross-section), but the relative positioning of the chromosomes within nuclei is largely random [59]. The tightly packed centromeric/pericentromeric portion (chromocenter) of every chromosome is often located close to the nuclear periphery or the nucleolus [4]. Chromocenters can fuse randomly, which produces strong inter-chromosomal interactions on the $\mathrm{Hi}-\mathrm{C}$ map. Telomeres often cluster around the nucleolus, except for those close to nucleolus-organizing regions (NORs) on chromosomes 2 and 4 (not shown in this diagram), which frequently associate with their respective chromocenters [4]. The interactive heterochromatic island (IHI)/KNOT engaged element (KEE) regions form strong intra-chromosomal, and sometimes inter-chromosomal contacts (not shown in this diagram), and are readily spotted on Hi-C maps 
regions have properties similar to those of sequences at the borders of animal TADs: there are limited chromatin contacts that cross these regions, and they are enriched for open chromatin and highly expressed genes [34], indicating a strong connection between transcription and local chromatin topology [33, 36]. These TAD-boundary-like and insulator-like regions were only noted after the resolution of A. thaliana $\mathrm{Hi}-\mathrm{C}$ maps was increased from the $20 \mathrm{~kb}$ to the $2 \mathrm{~kb}$ range [34]. This is reminiscent of studies on Saccharomyces cerevisiae, in which TADs were only noticed when utilizing a high-resolution variant of $\mathrm{Hi}-\mathrm{C}$ that uses DNA digestion by micrococcal nuclease instead of restriction enzymes [38].

Apart from more local interactions, there are prominent intra- and inter-chromosomal interactions among heterochromatic regions dispersed throughout the otherwise euchromatic chromosome arms [32-34, 39] (Fig. 1). These regions, named interactive heterochromatic islands (IHIs) [32] or KNOT engaged elements (KEEs) [33], range in size from $20-150 \mathrm{~kb}$ and are enriched for heterochromatic histone marks and transposons, even though they are not generally silenced. The mechanism by which these contacts are made remains unclear as similar features are found in other regions that do not behave as IHIs/KEEs. Interactions between IHIs/KEEs are largely unchanged even when most DNA methylation or heterochromatin H3K9me3 marks are removed [32]. These findings further suggest that DNA methylation and H3K9me2 do not directly cause the tethering of IHIs/KEEs.

The relationships between various chromatin modifications and chromatin packing have also been explored. H3K27me3, which is associated with Polycomb Repressive Complexes (PRCs), correlates with compact chromatin, and mutants lacking this histone mark have dramatically reduced chromatin contacts within such regions [32, 33]. H3K27me3 was also found to be enriched in 'positive strips', a special Hi-C feature apparent in a high-resolution A. thaliana Hi-C map [34]. Chromatin regions annotated as positive strips showed more frequent looping interaction with neighboring chromatin, thus forming contrasting lines of high contacts on the Hi-C map. These findings imply that, in addition to participating in local gene silencing, H3K27me3 might also directly or indirectly play a structural role in forming higher-order chromatin structure in plants.

\section{Challenges and outlook}

Plant genomes are very diverse, and so are their threedimensional (3D) structures [40]. A. thaliana has short chromosomes that adopt a rosette conformation. By contrast, species with long chromosomes feature what is known as the 'Rabl' conformation, and such differences are expected to be visible in Hi-C maps. Similarly, chromosomes can be quite differently organized, even in species that have similar chromosome number or genome size. The A. thaliana relatives Arabidopsis lyrata and Capsella rubella both have genomes that are about $50 \%$ larger than that of $A$. thaliana [41, 42]. However, while genome expansion occurred mostly on the chromosome arms in A. lyrata, the increase in genome size in C. rubella is confined to the centromeres. It will be interesting to see how these differences are reflected in $\mathrm{Hi}-\mathrm{C}$ maps of these species. Such closely related species that have rampant structural variation also afford a great opportunity to determine at a more fine-grained scale how deletions or insertions affect local chromatin-chromatin interactions.

The most impressive recent $\mathrm{Hi}-\mathrm{C}$ study was the one by Rao and colleagues [43], who provided an extremely high-resolution map of chromatin contacts in human cells, based on an enormous amount of DNA sequence. Similarly high-resolution $\mathrm{Hi}-\mathrm{C}$ maps are needed for $A$. thaliana, which has a very high gene density of about one gene per $5 \mathrm{~kb}$. If local chromatin loops are as widespread in A. thaliana as they are in humans, many chromatin loops that have roles in the regulation of transcription would have a comparatively small size. The identification of such small loops is a technically and computationally challenging task. First, conventional 3C-based methods need to be coupled with additional steps to increase the sequencing depth of query regions, as this is a prerequisite to achieving a more accurate estimate of background signals or random chromatin interactions that are associated with loci of interest. Approaches that can help to provide this resolution include selective amplification-based methods, such as $4 \mathrm{C}$ and $5 \mathrm{C}$ [44-46], the hybridizationbased $\mathrm{CHi}-\mathrm{C}$ method [47], and the immunoprecipitationbased ChIA-PET method [48]. Micro-C, which uses micrococcal nuclease to digest DNA into nucleosomes, further improves the resolution of contact maps [38]. On the computational side, re-evaluating the systematic biases of Hi-C experiments, as noted by Yaffe and Tanay [49], might be necessary for the robust detection of small chromatin loops. For example, besides being a factor that influences the amplification efficiency of library molecules, GC content has been shown to correlate with short-range chromatin contact in mammals, probably as a direct consequence of the action of certain GC-rich elements [50]. Other biases that confound the identification of chromatin loops over short genomic distances, such as the distribution of restriction enzyme cutting sites, must also be considered [34].

To complement sequencing-based methods, there are cytological tools that can visualize and monitor the behavior of chromatin loci in the nucleus. For example, padlock fluorescent in situ hybridization (FISH) [51] in combination with photoactivated localization microscopy (PALM) [52] might be able to increase the resolution of traditional FISH, so that small chromatin loops can be detected 
directly. There are already several live imaging systems that can be used to observe chromatin in plants. For example, visually trackable T-DNA insertions have revealed an influence of mobility and subnuclear localization on local gene expression [5]. In another study, physical clustering of trackable FLC-LacO transgene loci was observed in connection with Polycomb-mediated silencing [6]. Both studies employed $L a c O$ arrays that can be recognized specifically by bacterial LacI protein labeled with fluorescent proteins. Today, more sophisticated genome-editing techniques such as CRISPR/Cas9 would allow the non-random insertion of $\mathrm{LacO}$ arrays into the genome. A CRISPR/Cas-based chromatin-imaging method has already been used in mammalian cell lines for visualization of non-repetitive genomic loci [53]. The recent development of a multicolor CRISPR labeling system further allows simultaneous tracking of different loci [54].

Many environmental and developmental factors, such as light intensity, temperature, microbe infection, and cell differentiation, can trigger global rearrangement of chromatin in plants [55-58], and we are looking forward to studies that will complete the rather coarse picture we have today by analyzing local chromatin topology at high resolution under different conditions and in specific cell types. In addition, we are excited about the possibility of placing such observations in an evolutionary context, as plant genomes are particularly dynamic, undergoing frequent genome expansions and contractions over very short time scales. Surely, such dramatic changes in genome size must be reflected in the 3D organization of the genome itself. An important question will be whether chromatin loops and other types of interactions can compensate for drastic changes in the linear size of the genome, so that regulatory elements can exert their effects independently of whether they are 2 or $20 \mathrm{~kb}$ from a promoter.

\section{Abbreviations \\ 3C: Chromatin conformation capture; 3D: Three-dimensional; APOLO: AUXIN- REGULATED PROMOTER LOOP; crwn1: crowded nuclei 1; FISH: Fluorescent in situ hybridization; FLC: FLOWERING LOCUS C; FT: FLOWERING LOCUS T; IHI: Interactive heterochromatic island; KEE: KNOT engaged elements; IncRNA: long noncoding RNA; NFY: Nuclear Factor Y; PID: PINOID; RdDM: RNA-dependent DNA methylation; TAD: Topologically associating domain; TFL1: TERMINAL FLOWER 1; TSS: Transcription start site.}

\section{Competing interests}

$\mathrm{CL}$ and DW declare that they have no competing interests.

\section{Acknowledgements}

We wish to acknowledge the Max Planck Society and a Gottfried Wilhelm Leibniz Award from the Deutsche Forschungsgemeinschaft (DW), and the Marie Curie Fellowship PIIF-GA-2012-327608 (CL).

Published online: 21 August 2015

\section{References}

1. Dong F, Jiang J. Non-Rabl patterns of centromere and telomere distribution in the interphase nuclei of plant cells. Chromosome Res. 1998;6:551-8.

2. Rabl C. Über Zelltheilung Morphol Jahrb. 1885;10:214-330.
3. Fransz $\mathrm{P}$, de Jong H. From nucleosome to chromosome: a dynamic organization of genetic information. Plant J. 2011;66:4-17.

4. Fransz P, De Jong JH, Lysak M, Castiglione MR, Schubert I. Interphase chromosomes in Arabidopsis are organized as well defined chromocenters from which euchromatin loops emanate. Proc Natl Acad Sci U S A. 2002;99:14584-9.

5. Rosin FM, Watanabe N, Cacas JL, Kato N, Arroyo JM, Fang Y, et al. Genome-wide transposon tagging reveals location-dependent effects on transcription and chromatin organization in Arabidopsis. Plant J. 2008;55:514-25.

6. Rosa S, De Lucia F, Mylne JS, Zhu D, Ohmido N, Pendle A, et al. Physical clustering of $F L C$ alleles during Polycomb-mediated epigenetic silencing in vernalization. Genes Dev. 2013;27:1845-50.

7. Dixon JR, Selvaraj S, Yue F, Kim A, Li Y, Shen Y, et al. Topological domains in mammalian genomes identified by analysis of chromatin interactions. Nature. 2012;485:376-80.

8. Sexton T, Yaffe E, Kenigsberg E, Bantignies F, Leblanc B, Hoichman M, et al. Three-dimensional folding and functional organization principles of the Drosophila genome. Cell. 2012;148:458-72.

9. Hou C, Li L, Qin ZS, Corces VG. Gene density, transcription, and insulators contribute to the partition of the Drosophila genome into physical domains. Mol Cell. 2012;48:471-84.

10. Le TB, Imakaev MV, Mirny LA, Laub MT. High-resolution mapping of the spatial organization of a bacterial chromosome. Science. 2013;342:731-4.

11. Ay F, Bunnik EM, Varoquaux N, Bol SM, Prudhomme J, Vert JP, et al. Threedimensional modeling of the $P$. falciparum genome during the erythrocytic cycle reveals a strong connection between genome architecture and gene expression. Genome Res. 2014;24:974-88.

12. Mizuguchi T, Fudenberg G, Mehta S, Belton JM, Taneja N, Folco HD, et al. Cohesin-dependent globules and heterochromatin shape 3D genome architecture in S. pombe. Nature. 2014;516:432-5.

13. Gibcus JH, Dekker J. The hierarchy of the 3D genome. Mol Cell. 2013:49:773-82.

14. Sexton T, Cavalli G. The role of chromosome domains in shaping the functional genome. Cell. 2015;160:1049-59.

15. Louwers M, Bader R, Haring M, van Driel R, de Laat W, Stam M. Tissue- and expression level-specific chromatin looping at maize b1 epialleles. Plant Cell. 2009:21:832-42.

16. Crevillen P, Sonmez C, Wu Z, Dean C. A gene loop containing the floral repressor $F L C$ is disrupted in the early phase of vernalization. EMBO $\mathrm{J}$. 2013;32:140-8

17. Liu C, Teo ZW, Bi Y, Song S, Xi W, Yang X, et al. A conserved genetic pathway determines inflorescence architecture in Arabidopsis and rice. Dev Cell. 2013;24:612-22.

18. Cao S, Kumimoto RW, Gnesutta N, Calogero AM, Mantovani R, Holt 3rd BF. A distal CCAAT/NUCLEAR FACTOR Y complex promotes chromatin looping at the FLOWERING LOCUS T promoter and regulates the timing of flowering in Arabidopsis. Plant Cell. 2014;26:1009-17.

19. Liu L, Adrian J, Pankin A, Hu J, Dong X, von Korff M, et al. Induced and natural variation of promoter length modulates the photoperiodic response of FLOWERING LOCUS T. Nat Commun. 2014;5:4558.

20. Ariel F, Jegu T, Latrasse D, Romero-Barrios N, Christ A, Benhamed M, et al. Noncoding transcription by alternative RNA polymerases dynamically regulates an auxin-driven chromatin loop. Mol Cell. 2014;55:383-96.

21. letswaart R, Wu Z, Dean C. Flowering time control: another window to the connection between antisense RNA and chromatin. Trends Genet. 2012;28:445-53.

22. Ansari A, Hampsey M. A role for the CPF $3^{\prime}$-end processing machinery in RNAP II-dependent gene looping. Genes Dev. 2005;19:2969-78.

23. Laine JP, Singh BN, Krishnamurthy S, Hampsey M. A physiological role for gene loops in yeast. Genes Dev. 2009;23:2604-9.

24. Jegu T, Latrasse D, Delarue M, Hirt H, Domenichini S, Ariel F, et al. The BAF60 subunit of the SWI/SNF chromatin-remodeling complex directly controls the formation of a gene loop at FLOWERING LOCUS C in Arabidopsis. Plant Cell. 2014;26:538-51.

25. Swiezewski S, Liu F, Magusin A, Dean C. Cold-induced silencing by long antisense transcripts of an Arabidopsis Polycomb target. Nature. 2009:462:799-802.

26. Turck F, Roudier F, Farrona S, Martin-Magniette ML, Guillaume E, Buisine N, et al. Arabidopsis TFL2/LHP1 specifically associates with genes marked by trimethylation of histone H3 lysine 27. PLoS Genet. 2007;3, e86. 
27. Zhang X, Germann S, Blus BJ, Khorasanizadeh S, Gaudin V, Jacobsen SE. The Arabidopsis LHP1 protein colocalizes with histone H3 Lys27 trimethylation. Nat Struct Mol Biol. 2007;14:869-71

28. Turck F, Fornara F, Coupland G. Regulation and identity of florigen: FLOWERING LOCUS T moves center stage. Annu Rev Plant Biol. 2008;59:573-94.

29. Hou X, Zhou J, Liu C, Liu L, Shen L, Yu H. Nuclear factor Y-mediated H3K27me3 demethylation of the SOC1 locus orchestrates flowering responses of Arabidopsis. Nat Commun. 2014;5:4601.

30. Tiwari SB, Shen Y, Chang HC, Hou Y, Harris A, Ma SF, et al. The flowering time regulator CONSTANS is recruited to the FLOWERING LOCUS T promoter via a unique cis-element. New Phytol. 2010;187:57-66.

31. Kaufmann K, Wellmer F, Muino JM, Ferrier T, Wuest SE, Kumar V, et al. Orchestration of floral initiation by APETALA1. Science. 2010;328:85-9.

32. Feng S, Cokus SJ, Schubert V, Zhai J, Pellegrini M, Jacobsen SE. Genome-wide $\mathrm{Hi}-\mathrm{C}$ analyses in wild-type and mutants reveal high-resolution chromatin interactions in Arabidopsis. Mol Cell. 2014;55:694-707.

33. Grob S, Schmid MW, Grossniklaus U. Hi-C analysis in Arabidopsis identifies the KNOT, a structure with similarities to the flamenco locus of Drosophila. Mol Cell. 2014;55:678-93.

34. Wang C, Liu C, Roqueiro D, Grimm D, Schwab R, Becker C, et al. Genomewide analysis of local chromatin packing in Arabidopsis thaliana. Genome Res. 2015;25:246-56.

35. Grob S, Schmid MW, Luedtke NW, Wicker T, Grossniklaus U. Characterization of chromosomal architecture in Arabidopsis by chromosome conformation capture. Genome Biol. 2013;14:R129.

36. Lieberman-Aiden E, van Berkum NL, Williams L, Imakaev M, Ragoczy T, Telling $\mathrm{A}$, et al. Comprehensive mapping of long-range interactions reveals folding principles of the human genome. Science. 2009;326:289-93.

37. Gudynaite-Savitch L, Johnson DA, Miki BL. Strategies to mitigate transgenepromoter interactions. Plant Biotechnol J. 2009;7:472-85.

38. Hsieh TH, Weiner A, Lajoie B, Dekker J, Friedman N, Rando OJ. Mapping nucleosome resolution chromosome folding in yeast by Micro-C. Cell. 2015;162:108-19.

39. Moissiard G, Cokus SJ, Cary J, Feng S, Billi AC, Stroud H, et al. MORC family ATPases required for heterochromatin condensation and gene silencing. Science. 2012;336:1448-51.

40. Tiang $\mathrm{CL}, \mathrm{He}$ Y, Pawlowski WP. Chromosome organization and dynamics during interphase, mitosis, and meiosis in plants. Plant Physiol. 2012;158:26-34.

41. Hu TT, Pattyn P, Bakker EG, Cao J, Cheng JF, Clark RM, et al. The Arabidopsis lyrata genome sequence and the basis of rapid genome size change. Nat Genet. 2011;43:476-81.

42. Slotte T, Hazzouri KM, Agren JA, Koenig D, Maumus F, Guo YL, et al. The Capsella rubella genome and the genomic consequences of rapid mating system evolution. Nat Genet. 2013;45:831-5.

43. Rao SS, Huntley MH, Durand NC, Stamenova EK, Bochkov ID, Robinson JT, et al. A 3D map of the human genome at kilobase resolution reveals principles of chromatin looping. Cell. 2014;159:1665-80.

44. Dostie J, Richmond TA, Arnaout RA, Selzer RR, Lee WL, Honan TA, et al. Chromosome conformation capture carbon copy (5C): a massively parallel solution for mapping interactions between genomic elements. Genome Res. 2006:16:1299-309.

45. Simonis M, Klous $P$, Splinter E, Moshkin $Y$, Willemsen $R$, de Wit $E$, et al. Nuclear organization of active and inactive chromatin domains uncovered by chromosome conformation capture-on-chip (4C). Nat Genet. 2006:38:1348-54

46. Zhao Z, Tavoosidana G, Sjolinder M, Gondor A, Mariano P, Wang S, et al. Circular chromosome conformation capture (4C) uncovers extensive networks of epigenetically regulated intra- and interchromosomal interactions. Nat Genet. 2006;38:1341-7.

47. Schoenfelder S, Furlan-Magaril M, Mifsud B, Tavares-Cadete F, Sugar R, Javierre BM, et al. The pluripotent regulatory circuitry connecting promoters to their long-range interacting elements. Genome Res. 2015;25:582-97.

48. Li G, Fullwood MJ, Xu H, Mulawadi FH, Velkov S, Vega V, et al. ChIA-PET tool for comprehensive chromatin interaction analysis with paired-end tag sequencing. Genome Biol. 2010;11:R22.

49. Yaffe $\mathrm{E}$, Tanay A. Probabilistic modeling of $\mathrm{Hi}-\mathrm{C}$ contact maps eliminates systematic biases to characterize global chromosomal architecture. Nat Genet. 2011:43:1059-65.

50. Jin F, Li Y, Dixon JR, Selvaraj S, Ye Z, Lee AY, et al. A high-resolution map of the three-dimensional chromatin interactome in human cells. Nature. 2013;503:290-4.
51. Feng CM, Qiu Y, Van Buskirk EK, Yang EJ, Chen M. Light-regulated gene repositioning in Arabidopsis. Nat Commun. 2014;5:3027.

52. Schubert V, Weisshart K. Abundance and distribution of RNA polymerase II in Arabidopsis interphase nuclei. J Exp Bot. 2015;66:1687-98.

53. Chen B, Gilbert LA, Cimini BA, Schnitzbauer J, Zhang W, Li GW, et al. Dynamic imaging of genomic loci in living human cells by an optimized CRISPR/Cas system. Cell. 2013;155:1479-91.

54. Ma H, Naseri A, Reyes-Gutierrez P, Wolfe SA, Zhang S, Pederson T. Multicolor CRISPR labeling of chromosomal loci in human cells. Proc Natl Acad Sci U S A. 2015;112:3002-7.

55. Barneche F, Malapeira J, Mas P. The impact of chromatin dynamics on plant light responses and circadian clock function. J Exp Bot. 2014;65:2895-913.

56. Pecinka A, Dinh HQ, Baubec T, Rosa M, Lettner N, Mittelsten Scheid O. Epigenetic regulation of repetitive elements is attenuated by prolonged heat stress in Arabidopsis. Plant Cell. 2010;22:3118-29.

57. Pavet V, Quintero C, Cecchini NM, Rosa AL, Alvarez ME. Arabidopsis displays centromeric DNA hypomethylation and cytological alterations of heterochromatin upon attack by Pseudomonas syringae. Mol Plant Microbe Interact. 2006;19:577-87.

58. Tessadori F, Chupeau MC, Chupeau Y, Knip M, Germann S, van Driel R, et al. Large-scale dissociation and sequential reassembly of pericentric heterochromatin in dedifferentiated Arabidopsis cells. J Cell Sci. 2007;120:1200-8.

59. Pecinka A, Schubert V, Meister A, Kreth G, Klatte M, Lysak MA, et al. Chromosome territory arrangement and homologous pairing in nuclei of Arabidopsis thaliana are predominantly random except for NOR-bearing chromosomes. Chromosoma. 2004;113:258-69. 